\title{
Risk score model for the development of hepatocellular carcinoma in treatment-naïve patients receiving oral antiviral treatment for chronic hepatitis B
}

\author{
Won Sohn ${ }^{1, *}$, Ju-Yeon Cho ${ }^{2,}$, Ji Hoon Kim ${ }^{3}$, Jung Il Lee ${ }^{4}$, Hyung Joon $\mathrm{Kim}^{5}$, Min-Ah Woo, ${ }^{6,7}$, Sin-Ho Jung ${ }^{6,7}$, \\ and Yong-Han Paik ${ }^{7,8}$ \\ 'Department of Gastroenterology, Wonkwang University Sanbon Hospital, Gunpo; ${ }^{2}$ Department of Medicine, Chosun University \\ Hospital, Gwangju; ${ }^{3}$ Department of Internal Medicine, Korea University College of Medicine, Seoul; ${ }^{4}$ Department of Internal Medicine, \\ Yonsei University College of Medicine, Seoul; ${ }^{5}$ Department of Internal Medicine, Chung-Ang University College of Medicine, Seoul; \\ ${ }^{6}$ Statistics and Data Center, Research Institute for Future Medicine, Samsung Medical Center, Seoul; ${ }^{7}$ Department of Health Science \\ and Technology, SAIHST, Sungkyunkwan University, Seoul; ${ }^{8}$ Department of Medicine, Samsung Medical Center, Sungkyunkwan \\ University School of Medicine, Seoul, Korea
}

Background/Aims: This study aimed to develop and validate a risk prediction model for the development of hepatocellular carcinoma (HCC) in treatment-naïve patients receiving oral antiviral treatment for chronic hepatitis B (CHB).

Methods: We investigated 2,061 Korean treatment-naïve patients with CHB treated with entecavir as an initial therapy. A risk score model for $\mathrm{HCC}$ development was developed based on multivariable Cox regression model in a single center $(n=990)$ and was validated using the time-dependent area under the receiver operating characteristic curve (AUROC) in three other centers $(n=1,071)$. The difference of HCC development among risk groups (low, intermediate, and high) categorized by risk score was also investigated.

Results: The cumulative incidence rates of HCC at 5 years were $11.2 \%$ and $8.9 \%$ in the testing and validation cohorts, respectively. HCC-Risk Estimating Score in CHB patients Under Entecavir (HCC-RESCUE) is formulated as (age $+15 \times$ gender [female $=0 /$ male $=1]+23 \times$ cirrhosis [absence $=0 /$ presence $=1]$ ). The AUROCs at 1 year, 3 years, and 5 years were 0.82 , 0.81 , and 0.81 , respectively, in the validation cohort. A significant difference of $\mathrm{HCC}$ development in each risk group was determined by the 5 -year HCC risk score in the validation cohort (low risk group, 2.1\%; intermediate risk group, 9.3\%; high risk group, $41.2 \%, p<0.001$ ).

Conclusions: The study presents a new risk score model with a good ability to predict HCC development and determine high risk patients for HCC development consisting of readily available clinical factors in treatment-naïve CHB patients receiving entecavir. (Clin Mol Hepatol 2017;23:170-178)

Keywords: Chronic hepatitis B; Hepatocellular carcinoma; Assessment, Risk; Antiviral drugs

\begin{abstract}
Abbreviations:
AFP, alpha-fetoprotein; ALT, alanine transaminase; AST, aspartate transaminase: $\mathrm{CHB}$, chronic hepatitis $\mathrm{B} ; \mathrm{Cl}$, confidence interval; $\mathrm{CT}$, computed tomography; HBV, hepatitis B virus; HCC, hepatocellular carcinoma; HBeAg, hepatitis B virus envelope antigen; nucleos(t)ide, nucleoside/nucleotide; HBsAg, hepatitis B virus surface antigen; MR, magnetic resonance; MRI, magnetic resonance imaging; US, ultrasonography; SD, standard deviation.
\end{abstract}

Corresponding author: Yong-Han Paik

Department of Medicine, Samsung Medical Center, Sungkyunkwan University School of Medicine, 81 Irwon-ro, Gangnam-gu, Seoul 06351, Korea

Tel: +82-2-3410-3878, Fax: +82-2-3410-6983

E-mail:yh.paik@skku.edu

http://orcid.org/0000-0002-3076-2327 


\section{INTRODUCTION}

Hepatocellular carcinoma (HCC) mostly occurs in patients with chronic liver disease such as chronic hepatitis B (CHB) being the most common cause of HCC worldwide. The risk of HCC development increased 5 to 100 times in patients with CHB. Therefore, it is very important to predict the $\mathrm{CHB}$ patients with a high risk of HCC development and to carry out an active surveillance for HCC detection in high risk groups.

Oral antiviral agents are now a mainstay of the treatment in CHB patients. In particular, entecavir and tenofovir, which show excellent viral suppression and little drug resistance, are recommended as an initial therapy for CHB patients. ${ }^{2,3}$ Long-term treatment of entecavir decreases the risk of HCC development in patients with CHB compared to untreated patients. ${ }^{4,5}$ However, oral antiviral treatment can not completely eradicate the risk of HCC development in CHB patients. Our previous study showed that CHB patients treated with oral antiviral agents still have a higher risk of $\mathrm{HCC}$ development than those at an inactive state of $\mathrm{CHB}^{6}{ }^{6}$ Therefore, it is important to predict the risk of HCC development in CHB patients receiving antiviral treatment.

Most of the previously reported prediction models for HCC development included $\mathrm{CHB}$ patients without antiviral treatment. ${ }^{7-9} \mathrm{~A}$ recent study presented a prediction model for HCC development in CHB patients who were treated with either entecavir or tenofovir. ${ }^{10}$ However, this study included a fair number of patients who were treatment-experienced with interferon or nucleos(t)ide analogs (NAs) before entecavir or tenofovir treatment. At present, there is no risk model to predict HCC development in treatment-naive CHB patients receiving oral antiviral treatment as an initial therapy.

Our study aimed to establish and validate a risk prediction model for HCC development in treatment-naïve CHB patients receiving entecavir to identify the risk of progression to HCC in patients on NA treatment. All patients enrolled in this study were treated with entecavir for CHB as an initial therapy in both the testing and validation groups. Furthermore, the high risk group for HCC development in CHB patient on entecavir was also determined and validated.

\section{PATIENTS AND METHODS}

\section{Study design \& population}

This study retrospectively analyzed 2,061 treatment-naïve CHB patients treated with entecavir as an initial therapy from 4 centers in Seoul, Korea. The criteria for the use of entecavir were based on the Korean Association for the Study of the Liver (KASL) guideline: (i) hepatitis $B$ virus (HBV) DNA $\geq 20,000 \mathrm{IU} / \mathrm{mL}$ with serum aspartate transaminase (AST) or alanine transaminase (ALT) level $\geq 2$ upper limit of normal (ULN) in HBeAg-positive patients; (ii) HBV DNA $\geq 2,000 \mathrm{IU} / \mathrm{mL}$ with serum AST or ALT level $\geq 2 \mathrm{ULN}$ in HBeAg-negative patients; and (iii) HBV DNA $\geq 2,000 \mathrm{IU} / \mathrm{mL}$ with serum AST or ALT level above ULN in liver cirrhosis. ${ }^{11,12}$ CHB was defined as HBsAg positive for more than 6 months. The population was divided into two groups: a testing cohort (Sungkyunkwan University Samsung Medical (enter, $\mathrm{n}=990$ ) and a validation cohort (Chung-Ang University Hospital, Yonsei University Gangnam Severance Hospital, and Korea University Guro Hospital, $n=1,061)$. The testing cohort was used to assess risk factors for HCC development and establish the HCC risk prediction model. The validation cohort was used to verify the risk prediction model for HCC development.

Inclusion criteria were age $\geq 18$ years and treatment with entecavir as an initial therapy for CHB. Exclusion criteria consisted of co-infection with hepatitis $\mathrm{C}$ virus, prior treatment history of NAs or interferon, entecavir administration less than 6 months, HCC development within 6 months after starting entecavir, loss of follow-up within 6 months after starting entecavir, liver transplantation without HCC development after starting entecavir, and incomplete laboratory data. Liver cirrhosis was defined based on liver histology or ultrasonographic findings (coarse parenchymal echogenicity with irregular surface nodularity) with/without the manifestation of portal hypertension (splenomegaly, ascites, gastroesophageal varices, and hepatic encephalopathy).

\section{Assessment of outcome and follow-up}

All the patients were assessed with AST, ALT, platelet count, serum albumin, the presence of HBV envelope antigen (HBeAg), alpha-fetoprotein (AFP) and ultrasonography at baseline. To exclude HCC at baseline, contrast-enhanced computed CT (CT) or magnetic resonance imaging (MRI) was additionally performed in high risk group for HCC development: i) hepatic mass in ultrasonography, ii) the suspicion of regenerative nodule or dysplastic nodule with advanced LC in ultrasonography, iii) increased serum AFP, and iv) family history of HCC. Pre-treatment serum HBV DNA level was measured using COBAS TaqMan quantitative test for hepatitis B virus (Roche Molecular Systems Inc., Branchburg, NJ, USA). After starting entecavir, the patients were checked for serum ami- 
notransferase level, status of HBeAg, and HBV DNA level every 3 or 6 months. The outcome of this study is HCC development in CHB patients receiving entecavir. For HCC surveillance, enrolled patients underwent radiologic study (ultrasonography and/or contrast-enhanced (T) with serum AFP every 6 months. In addition, MRI or liver biopsy was performed to confirm the diagnosis of HCC if necessary. HCC was diagnosed according to the American Association for the Study of Liver Diseases (AASLD) guideline. ${ }^{13}$ Time to HCC development was defined as the interval between the initiation of entecavir treatment and the detection of HCC. The follow-up duration was defined as the period between the initiation of entecavir and either HCC development or the last visit to outpatient clinic before December 31, 2014.

\section{Statistical analysis}

Categorical variables were presented as frequencies with percentages. Continuous variables were described as a mean value with standard deviation (SD) and a median value with interquartile range according to parametric and non-parametric distributions, respectively. From the testing set, a risk prediction model for the time to HCC was developed using a Cox regression model. For variable selection, the stepwise procedure with inclusion and exclusion criteria using 2-sided alpha $=5 \%$ was used. Based on the relative size of the regression estimates in the fitted prediction model, a HCC risk score model was developed. To confirm that this risk score is applicable to both data sets, the distribution of the time-dependent area under receiver operating characteristic curve (AUROC) in each data set using 1,000 bootstrap samples was estimated and compared between the two sets. The cutoff values of the fitted risk score for $<5 \%$ (low risk group), $5-20 \%$ (intermediate risk group), $>20 \%$ (high risk group) of 5 -year $\mathrm{HCC}$ probability was identified, and generated a time to HCC probability curve for the both data sets. Applying these cutoffs to the validation set, the time to HCC distribution was compared among the three risk groups using the log-rank test. A plot of 5-year HCC probabilities for the fitted risk scores was also generated. These analyses were conducted using SAS version 9.4 (SAS Institute, Cary, NC, USA) and R 3.2.3 (Vienna, Austria; http://www.R-project.org).

\section{RESULTS}

\section{Baseline characteristics and HCC development}

Baseline characteristics of the testing and validation cohorts are demonstrated in Table 1. The validation cohort had higher levels of platelet count, AST, ALT, pre-treatment $\log _{10} \mathrm{HBV}$ DNA, and a higher proportion of HBeAg positivity than the testing cohort

Table 1. Baseline characteristics of all patients $(n=2,061)$

\begin{tabular}{|c|c|c|c|}
\hline & Testing cohort $(n=990)$ & Validation cohort $(n=1,071)$ & $P$-value \\
\hline Age (years) & $47.4 \pm 10.5$ & $46.6 \pm 11.5$ & 0.096 \\
\hline Gender (n, \%) & & & 0.282 \\
\hline Female & $349(35)$ & $402(37)$ & \\
\hline Male & $641(65)$ & $669(63)$ & \\
\hline Platelet $\left(\times 10^{3} / \mathrm{mm}^{3}\right)$ & $152.8 \pm 62.8$ & $161.8 \pm 69.9$ & 0.002 \\
\hline Albumin (g/dL) & $4.0 \pm 0.5$ & $3.9 \pm 0.6$ & 0.017 \\
\hline AST (U/L) & $113.1 \pm 185.2$ & $184.6 \pm 282.5$ & $<0.001$ \\
\hline ALT (U/L) & $156.1 \pm 295.0$ & $234.4 \pm 358.8$ & $<0.001$ \\
\hline $\log _{10} H B V$ DNA (IU/mL) & $6.5 \pm 1.4$ & $6.6 \pm 1.5$ & $<0.001$ \\
\hline HBeAg $(n, \%)$ & & & 0.015 \\
\hline Negativity & $434(44)$ & $413(39)$ & \\
\hline Positivity & $556(56)$ & $658(61)$ & \\
\hline \multicolumn{4}{|l|}{ Liver cirrhosis (n, \%) } \\
\hline Absence & $601(61)$ & $695(65)$ & 0.049 \\
\hline Presence & $389(39)$ & $376(35)$ & \\
\hline
\end{tabular}

Data are present as the mean \pm standard deviation or number of patients (percentages in parentheses).

AST, aspartate aminotransferase; ALT, alanine aminotransferase; HBV, hepatitis B virus; HBeAg, hepatitis B envelope antigen. 
$(P<0.05)$. The level of albumin, and the proportion of liver cirrhosis were higher in the testing cohort compared to the validation cohort $(P<0.05)$. There was no significant difference in age or gender between the two cohorts.

The median duration of follow-up was 2.1 (interquartile range: 1.3-4.2) years in the testing cohort and 3.5 (interquartile range: 2.4-4.9) years in the validation cohort. HCC development was observed in $58(5.9 \%)$ patients in the testing cohort and 85 $(6.3 \%)$ patients in the validation cohort. Figure 1 shows the cumulative incidence rates of HCC in the testing and validation cohorts. The cumulative incidence rates of HCC at 1 year, 3 years,

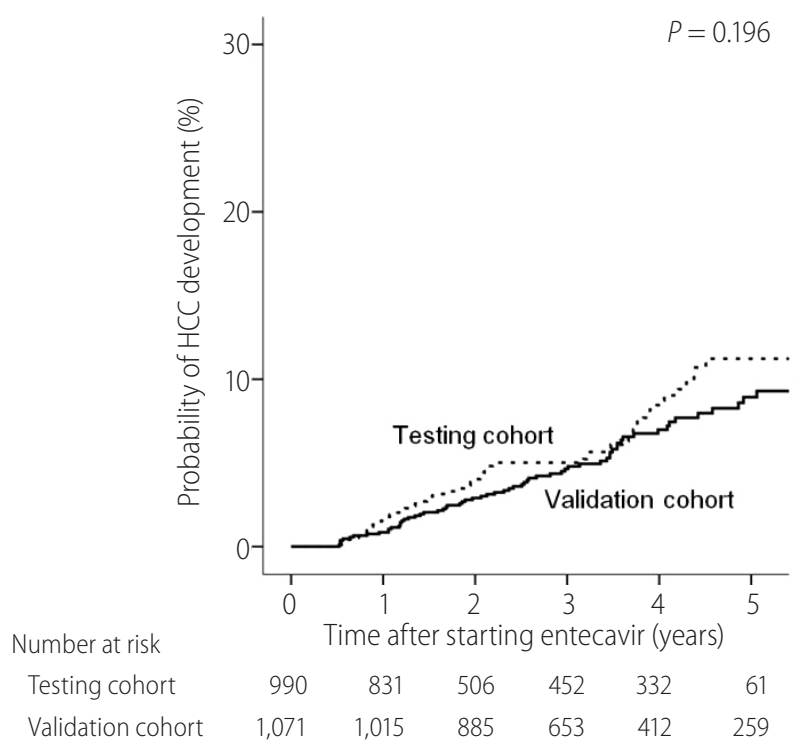

Figure 1. Cumulative incidence of HCC development in the testing and validation cohorts. HCC, hepatocellular carcinoma. and 5 years are $1.5 \%, 5.0 \%$, and $11.2 \%$, respectively, in the testing cohort and $0.9 \%, 4.6 \%$, and $8.9 \%$, respectively, in the validation cohort. There was no significant difference in HCC development between the two cohorts $(P=0.196)$.

\section{Risk factors for HCC development in patients receiving entecavir for $\mathrm{CHB}$}

To establish the risk prediction model for HCC, the risk factors for HCC development were analyzed in the testing cohort (Table 2). Univariable analysis indicated that HCC development was associated with age (hazard ratio [HR] 1.08, $P<0.001$ ), platelet count (HR 0.99, $P<0.001$ ), albumin level (HR 0.51, $P=0.004$ ), ALT level (HR 0.99, $P=0.001$ ), pre-treatment $\log _{10} \mathrm{HBV}$ DNA (HR 0.80, $P=0.014)$, and liver cirrhosis (HR 7.34, $P<0.001)$. Multivariable analysis showed that the risk factor for HCC development was age (HR 1.08, $P<0.001)$, male (HR 2.89, $P=0.001)$, and liver cirrhosis (HR 5.26, $P<0.001$ )

The difference of HCC development according to virological response to entecavir was evaluated in the testing cohort. Complete virological response (CVR) group $(n=933)$ was defined as continuous virological suppression (HBV DNA $<69 \mathrm{IU} / \mathrm{mL}$ ) while incomplete virological response (IVR) group $(n=57)$ was defined as HBV DNA fluctuation above $\geq 69 \mathrm{IU} / \mathrm{mL}$. HCC development was observed in 57 patients from CVR group and in 1 patient from IVR group. The mean durations of time to HCC development were 5.58 (95\% Cl: 5.49-5.67) and 5.11 (95\% Cl: 4.42-5.81) years in CVR and IVR group, respectively. There is no statistical difference in HCC development according to virological response $(P=0.703)$.

Table 2. Univariable and multivariable analyses of risk factors for HCC development in the testing cohort $(n=990)$

\begin{tabular}{|c|c|c|c|c|c|}
\hline & $\begin{array}{c}\text { Univariable HR } \\
(95 \% \mathrm{Cl})\end{array}$ & $P$-value & $\begin{array}{c}\text { Multivariable HR } \\
(95 \% \mathrm{Cl})\end{array}$ & $P$-value & $\beta$-coefficient \\
\hline Age (years) & $1.08(1.05-1.11)$ & $<0.001$ & $1.08(1.04-1.11)$ & $<0.001$ & 0.073 \\
\hline Men & $1.54(0.85-2.77)$ & 0.151 & $2.89(1.55-5.41)$ & 0.001 & 1.062 \\
\hline Platelet $\left(\times 10^{3} / \mathrm{mm}^{3}\right)$ & $0.99(0.98-0.99)$ & $<0.001$ & & & \\
\hline Albumin (g/dL) & $0.51(0.32-0.80)$ & 0.004 & & & \\
\hline AST (U/L) & $1.00(0.99-1.00)$ & 0.161 & & & \\
\hline $\mathrm{ALT}(\mathrm{U} / \mathrm{L})$ & $0.99(0.99-1.00)$ & 0.001 & & & \\
\hline $\log _{10} \mathrm{HBV}$ DNA (IU/mL) & $0.80(0.67-0.96)$ & 0.014 & & & \\
\hline HBeAg positivity & $0.77(0.46-1.29)$ & 0.328 & & & \\
\hline Liver cirrhosis & $7.34(3.61-14.94)$ & $<0.001$ & $5.26(2.55-10.84)$ & $<0.001$ & 1.659 \\
\hline
\end{tabular}

HCC, hepatocellular carcinoma; HR, hazard ratio; $\mathrm{Cl}$, confidence interval; AST, aspartate aminotransferase; ALT, alanine aminotransferase; HBV, hepatitis B virus; HBeAg, hepatitis B envelope antigen. 
Table 3. Time-dependent area under the receiver operating characteristic curve (AUROC) for HCC development using the HCC-RESCUE model in the testing and validation cohorts

\begin{tabular}{llllll}
\hline & \multicolumn{1}{c}{ 1-year } & \multicolumn{1}{c}{ 2-year } & \multicolumn{1}{c}{ 3-year } & 4-year & \multicolumn{1}{c}{ 5-year } \\
\hline Testing cohort $(n=990)$ & $0.798(0.752-0.843)$ & $0.789(0.747-0.831)$ & $0.788(0.746-0.830)$ & $0.786(0.743-0.829)$ & $0.768(0.715-0.820)$ \\
Validation cohort $(n=1,071)$ & $0.817(0.775-0.858)$ & $0.817(0.776-0.858)$ & $0.810(0.769-0.851)$ & $0.806(0.763-0.850)$ & $0.809(0.764-0.854)$ \\
$P$-value & 0.273 & 0.172 & 0.232 & 0.259 & 0.123 \\
\hline
\end{tabular}

The value is AUROC with $95 \%$ confidence interval at each year. $P$-value means the statistical difference of AUROC at each year between the testing and validation cohorts.

HCC, hepatocellular carcinoma; HCC-RESCUE, HCC-Risk Estimating Score in chronic hepatitis B patients under entecavir.

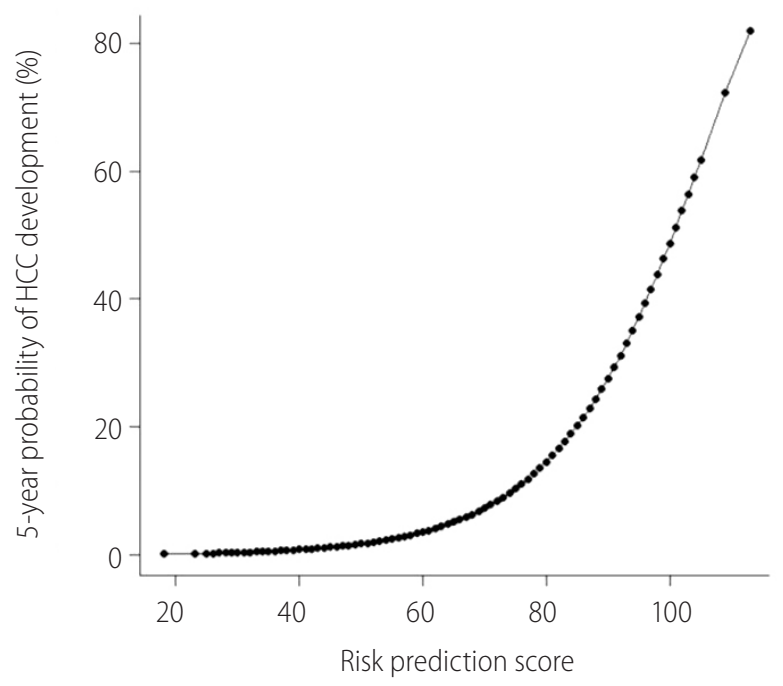

Figure 2. Five-year cumulative probability of HCC development in the testing cohort according to HCC-RESCUE score. HCC, hepatocellular carcinoma; HCC-RESCUE, HCC-Risk Estimating Score in chronic hepatitis B patients under entecavir.

\section{Testing and validation of the risk score model}

Based on the significant risk factors (age, gender, and liver cirrhosis) presented in the multivariable analysis, a new risk score as a prediction model for HCC development in CHB patients receiving entecavir was established and applied to the testing and validation cohorts. The risk score based on the above model is formulated as (age $+15 \times$ gender [female $=0 /$ male $=1]+23 \times$ cirrhosis [absence $=0 /$ presence $=1]$ ). We named it as HCC-Risk Estimating Score in CHB patients Under Entecavir (HCC-RESCUE). The timedependent AUROC using the new risk score was assessed to predict HCC risk in the testing and validation cohorts (Table 3). The AUROCs at 1 year, 2 years, 3 years, 4 years, and 5 years were 0.798 (95\% Cl: $0.752-0.843), 0.789$ (95\% Cl: $0.747-0.831)$, 0.788 (95\% Cl: $0.746-0.830), 0.786$ (95\% Cl: $0.743-0.829)$, and
0.817 (95\% Cl: $0.715-0.821)$, respectively, in the testing cohort and 0.817 (95\% Cl: $0.775-0.858), 0.817$ (95\% Cl: $0.777-0.858)$, 0.810 (95\% Cl: $0.769-0.851), 0.806$ (95\% Cl: $0.763-0.850)$,and 0.809 (95\% Cl: $0.764-0.854)$, respectively, in the validation cohort. There was no significant difference in AUROC at each year between the two cohorts $(P=0.273, P=0.172, P=0.232, P=0.259$, and $P=0.123$ ). Figure 2 shows 5 -year cumulative probability of HCC development in CHB patients receiving entecavir according to the increase of HCC-RESCUE score in the testing cohort was calculated. The cumulative probability of HCC development at a certain year $(t)$ is formulated as follows: $t$-year HCC probability = $1-S(t) . S(t)=\exp \left\{-H_{0}(t) \exp (0.07 \times\right.$ HCC-RESCUE score) $\left.)\right\} . S(t)$ means $t$-year HCC-free probability. Five-year cumulative probability of HCC development according to each risk score point is presented in Supplementary Table 1. Five-year cumulative probabilities of HCC development in patients with HCC-RESCUE scores of $20,40,60,80$, and 100 points were $0.2 \%, 0.8 \%, 3.6 \%, 14.5 \%$, and $48.7 \%$, respectively.

\section{HCC development according to risk groups based on the new risk score}

Patients were categorized using the new HCC-RESCUE risk score. The patients were categorized into three risk groups according to the 5-year cumulative probability of HCC development: low risk group, <5\%; intermediate risk group, 5-20\%; high risk group, $\geq 20 \%$. The difference in HCC development by each risk group was assessed in the testing and validation cohorts (Fig. 3). The minimum and maximum values of the new risk score were 18 and 113 points. The equivalent risk scores to $5 \%$ and $20 \%$ of 5 -year cumulative probability of HCC development were 65 and 85 points, respectively. Therefore, the categorization of the risk groups using the HCC-RESCUE scores is present as the following: low risk group, $\leq 64$ points; intermediate risk group, 65-84 points; high risk group, $\geq 85$ points. 

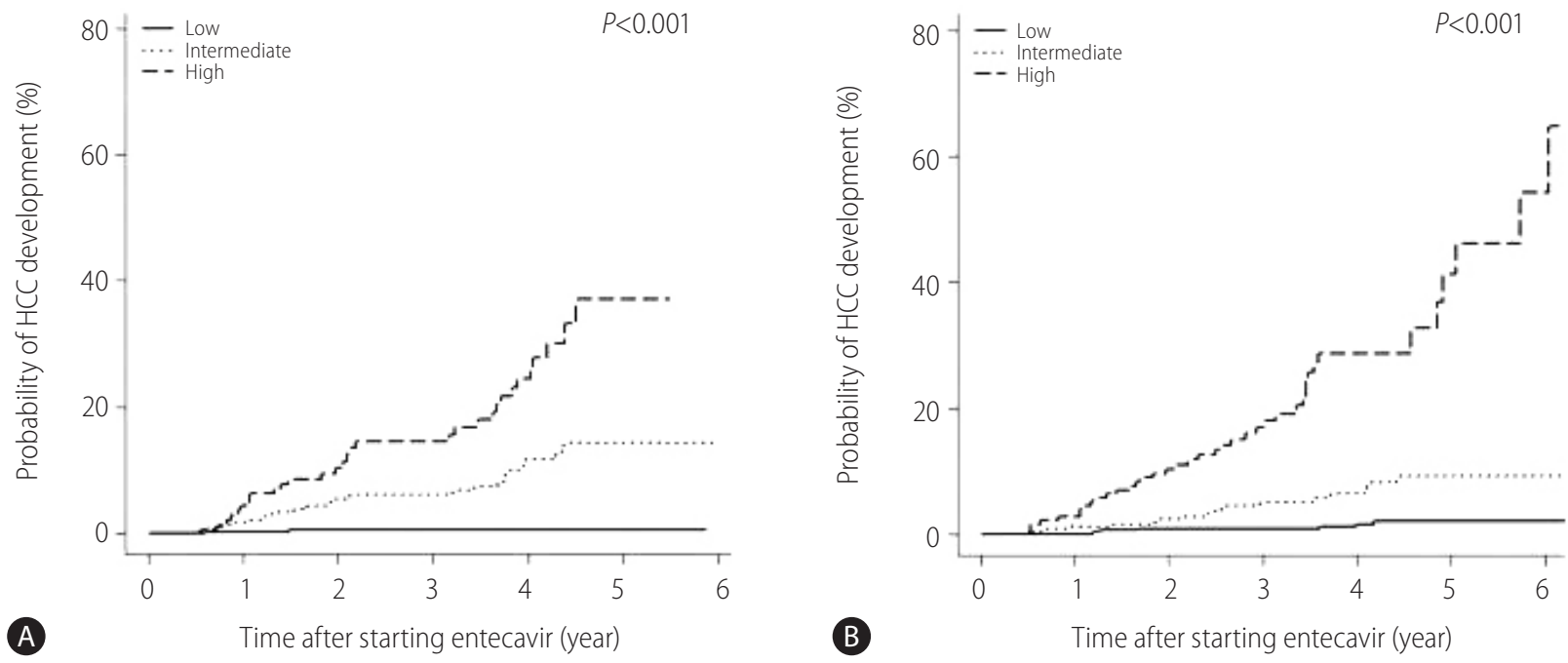

Figure 3. Cumulative probability of HCC in the testing (A) and validation (B) cohorts according to risk group based on HCC-RESCUE sCore (low risk group: score $\leq 64$, intermediate risk group: score 65-84, and high risk group: score $\geq 85$ ). HCC, hepatocellular carcinoma; HCC-RESCUE, HCC-Risk Estimating Score in chronic hepatitis B patients under entecavir.

In the testing cohort, the cumulative probabilities of HCC development at 1 year, 3 years, and 5 years were $0.2 \%, 0.5 \%$, and $0.5 \%$, respectively, in the low risk group, $1.8 \%, 6.2 \%$ and $14.4 \%$, respectively, in the intermediate risk group and $4.4 \%, 14.6 \%$ and $37.1 \%$, respectively, in the high risk group (Fig. 3A). There was a significant difference in $\mathrm{HCC}$ development according to the risk groups $(P<0.001)$. In the validation cohort, the cumulative probabilities of $\mathrm{HCC}$ development at 1 year, 3 years, and 5 years were $0 \%, 0.9 \%$, and $2.1 \%$, respectively, in the low risk group, $1.3 \%$, $5.2 \%$, and $9.3 \%$, respectively, in the intermediate risk group and $2.8 \%, 17.1 \%$ and $41.2 \%$, respectively, in the high risk group (Fig. 3B). There was a significant difference in HCC development according to the risk groups $(p<0.001)$.

\section{DISCUSSION}

We present herein HCC-RESCUE, a new model predicting the risk of $\mathrm{HCC}$ development optimized in treatment-naïve CHB patients receiving oral antiviral agent. The HCC risk score model was developed using treatment-naïve CHB patients on entecavir from a single tertiary care center and externally validated by patients from 3 university-affiliated hospitals. The difference in probability of HCC development according to the risk groups categorized by HCC-RESCUE was also verified.

Oral antiviral treatment using NA has a pivotal role in preventing HCC development in $\mathrm{CHB}{ }^{14}$ The use of potent oral antiviral treatment can effectively suppress HBV replication in patients with CHB. However, our previous study demonstrated that the risk of HCC development in patients treated with oral antiviral agent is still significantly higher compared to patients at an inactive state of CHB. ${ }^{6}$ Thus, oral antiviral treatment reduces but not eliminates the risk of developing $\mathrm{HCC}$ in CHB patients. Therefore, it is definitely required to establish a risk prediction model for HCC development in patients receiving antiviral treatment for $\mathrm{CHB}$.

There have been several studies that developed a $\mathrm{HCC}$ risk model in patients with $\mathrm{CHB} .{ }^{7-10,15}$ However, most of the models were derived from CHB patients without experience of antiviral treatment ${ }^{7-9}$ or included a very minor proportion $(15.1 \%)$ of patients receiving antiviral therapy. ${ }^{15}$ Previous risk models suggest various risk factors for HCC development in CHB patients. Age, albumin, bilirubin, cirrhosis and HBV DNA level were the risk factors for HCC development from a Hong Kong cohort. ${ }^{7}$ Another risk model from Hong Kong indicated the risk factors for HCC development as age, gender, basal core promoter mutation, cirrhosis, and HBV DNA level. ${ }^{8}$ Yang et al. report age, gender, ALT, HBeAg, and HBV DNA level as the risk factors for HCC development. ${ }^{9}$ In the study of Wong et al. age, albumin, HBV DNA level, and liver stiffness measurement using transient elastrography were considered as the risk factors of HCC development. ${ }^{15}$

Compared to previous studies, the risk factors in our model simply consist of age, gender, and cirrhosis. The main difference between our study and the previous studies is that our risk model does not include virological (pre-treatment HBV DNA level and 
HBeAg) or biochemical (ALT, albumin, and bilirubin) factors. The difference in the risk factors for $\mathrm{HCC}$ development may be caused by the characteristics of the enrolled patients in the study, a critical factor being treatment with an antiviral agent. As mentioned above, previous risk models were derived from CHB patients without antiviral treatment or included a small portion of patients receiving antiviral treatment. In contrast, our risk model consists purely of treatment-naïve patients receiving entecavir for $\mathrm{CHB}$ in both the testing and validation cohorts.

A large-scale Taiwanese cohort study revealed that HBV DNA level is an independent risk factor for HCC development in CHB without antiviral treatment. ${ }^{16}$ However, HBV DNA level is no longer a risk factor for HCC development in CHB patients receiving antiviral treatment. ${ }^{4,6,17-19}$ Although the HBV DNA level was included in previous risk models for HCC development, the level of HBV DNA was not a risk factor for HCC development in our study. This may be due to the fact that our study cohort includes only patients on entecavir treatment. The study suggests that the risk of HCC development in CHB patients receiving antiviral treatment is not increased by baseline factors, pre-treatment HBV DNA and liver function tests, modifiable by treatment. Only pre-treatment baseline factors which are difficult to modify with antiviral treatment such as age, gender, and liver cirrhosis are remained as the risk factors for HCC development. The effect of regression of cirrhosis by long-term use of entecavir or tenofovir on the risk of HCC development needs to be further studied. ${ }^{20,21}$

The HCC-RESCUE includes three readily available clinical factors with a good predictability for HCC development in the validation cohort (time-dependent AUROC >0.8). Some studies validated the risk of HCC development in CHB patients receiving oral antiviral treatment using previous risk score models with favorable predictabilities. ${ }^{22,23}$ However, the majority of the patients in those studies were treatment experienced with either pegylated interferon or nucleos(t)ide analogue other than entecavir or tenofovir. Our study also showed that the HCC-RESCUE model has a good ability to determine the high risk group for HCC development in CHB patients receiving antiviral treatment. There was a significant difference in HCC development between the low, intermediate, and high risk groups that were categorized based on the HCCRESCUE score.

All the patients in this study were of Asian ethnicity and were treated with entecavir as an initial therapy without any previous antiviral treatment. The maximum value of the risk of HCC development at 5-year was higher in our model compared to Caucasian model $(82 \%$ vs. $47 \%) .^{10}$ This finding may be caused by the differ- ence in HCC incidence according to HBV genotype. Almost all patients with CHB (98\%) have genotype C in Korea which is known to have a higher risk of HCC development compared to other genotypes. ${ }^{24,25}$ Therefore, this risk score model may have a higher specificity in Asian patients who are abundant with genotype $C$ than Caucasian patients. The risk score needs to be further validated in patients of other ethnic origin.

The goals of antiviral treatment are the prevention of HCC as well as the reduction of liver-related mortality. ${ }^{2,3}$ However, the risk of HCC still remains high despite antiviral treatment. ${ }^{6}$ Thus, it is crucial to determine the high risk groups for $\mathrm{HCC}$ development in patients receiving antiviral treatment. Most guidelines recommend HCC surveillance with ultrasonography and/or serum AFP every six months for high risk group in chronic liver disease. ${ }^{13,26}$ The benefit of performing more aggressive surveillance using other imaging modalities (i.e. CT or MR imaging) or using shorter interval (less than 6 months) for very high risk groups is not clear. Our risk score model could aid in determining the high risk group for HCC development and further individualize the surveillance strategy in CHB patients receiving antiviral treatment.

Our study is limited by the retrospective nature of the study conducted based on historical cohorts from four tertiary care centers. We could not study the patients' compliance of entecavir administration. Although there may be differences in the treatment pattern or protocol among the four centers, the influence on the study are minimal because the initiation or continuation of antiviral treatment for CHB in Korea is based on the KASL guideline and regulated by government-based mandatory health insurance system. ${ }^{11,12}$ Also, although tenofovir is a potent agent, this study included CHB patients treated only with entecavir due to the delayed release of tenofovir in Korea (December, 2012).

In conclusion, the results of this study represent a new risk model HCC-RESCUE consisting of readily available clinical factors, age, gender and liver cirrhosis, in treatment-naïve CHB patients receiving entecavir. HCC-RESCUE accurately predicts the risk of HCC development and can guide clinicians in determining the high risk group for HCC development in patients receiving oral antiviral agents.

\section{Financial support}

This study was supported by The Research Supporting Program of The Korean Association for the Study of the Liver and The Korean Liver Foundation (KASL-KLF 2014-03). 


\section{Author's contributions}

Dr. Sohn, Dr. Cho, and Dr. Paik have full access to all of the data in the study and take responsibility for the integrity of the data and the accuracy of the data analysis.

-Study concept and design: Sohn, Cho, and Paik.

-Acquisition of data: Cho, JH Kim, Lee, and HJ Kim.

-Analysis and interpretation of data: Sohn, Cho, JH Kim, Lee, HJ Kim, and Paik.

-Drafting of the manuscript: Sohn, Cho, Woo, Jung, and Paik.

-Critical revision of the manuscript for important intellectual content: JH Kim, Lee, and HJ Kim.

-Statistical analysis: Sohn, Cho, Woo, Jung, and Paik.

\section{Conflicts of Interest}

The authors have no conflicts to disclose.

\section{SUPPLEMENTARY MATERIALS}

Supplementary materials are available at Clinical and Molecular Hepatology website (http://www.e-cmh.org).

\section{REFERENCES}

1. El-Serag HB. Epidemiology of viral hepatitis and hepatocellular carcinoma. Gastroenterology 2012;142:1264-1273.e1.

2. European Association For The Study Of The Liver. EASL clinical practice guidelines: management of chronic hepatitis B virus infection. J Hepatol 2012;57:167-185.

3. Terrault NA, Bzowej NH, Chang KM, Hwang JP, Jonas MM, Murad $\mathrm{MH}$, et al. AASLD guidelines for treatment of chronic hepatitis $\mathrm{B}$. Hepatology 2016;63:261-283.

4. Hosaka T, Suzuki F, Kobayashi M, Seko Y, Kawamura Y, Sezaki H, et al. Long-term entecavir treatment reduces hepatocellular carcinoma incidence in patients with hepatitis B virus infection. Hepatology 2013;58:98-107.

5. Wong GL, Chan HL, Mak CW, Lee SK, Ip ZM, Lam AT, et al. Entecavir treatment reduces hepatic events and deaths in chronic hepatitis B patients with liver cirrhosis. Hepatology 2013;58:1537-1547.

6. Cho JY, Paik YH, Sohn W, Cho HC, Gwak GY, Choi MS, et al. Patients with chronic hepatitis $B$ treated with oral antiviral therapy retain a higher risk for HCC compared with patients with inactive stage disease. Gut 2014;63:1943-1950.

7. Wong VW, Chan SL, Mo F, Chan TC, Loong HH, Wong GL, et al. Clinical scoring system to predict hepatocellular carcinoma in chron- ic hepatitis B carriers. J Clin Oncol 2010;28:1660-1665.

8. Yuen MF, Tanaka Y, Fong DY, Fung J, Wong DK, Yuen JC, et al. Independent risk factors and predictive score for the development of hepatocellular carcinoma in chronic hepatitis B. J Hepatol 2009;50:8088.

9. Yang HI, Yuen MF, Chan HL, Han KH, Chen PJ, Kim DY, et al. Risk estimation for hepatocellular carcinoma in chronic hepatitis B (REACH$\mathrm{B})$ : development and validation of a predictive score. Lancet Oncol 2011;12:568-574.

10. Papatheodoridis G, Dalekos G, Sypsa V, Yurdaydin C, Buti M, Goulis J, et al. PAGE-B predicts the risk of developing hepatocellular carcinoma in caucasians with chronic hepatitis B on 5-year antiviral therapy. J Hepatol 2016;64:800-806.

11. Korean Association for the Study of the Liver. KASL clinical practice guidelines: management of chronic hepatitis B. Clin Mol Hepatol 2012;18:109-162.

12. Korean Association for the Study of the Liver. KASL clinical practice guidelines: management of chronic hepatitis B. Clin Mol Hepatol 2016;22:18-75.

13. Bruix J, Sherman M; Practice Guidelines Committee, American Association for the Study of Liver Diseases. Management of hepatocellular carcinoma. Hepatology 2005;42:1208-1236.

14. Papatheodoridis GV, Chan HL, Hansen BE, Janssen HL, Lampertico P. Risk of hepatocellular carcinoma in chronic hepatitis B: assessment and modification with current antiviral therapy. J Hepatol 2015;62:956-967.

15. Wong GL, Chan HL, Wong CK, Leung C, Chan CY, Ho PP, et al. Liver stiffness-based optimization of hepatocellular carcinoma risk score in patients with chronic hepatitis B. J Hepatol 2014;60:339-345.

16. Chen CJ, Yang HI, Su J, Jen CL, You SL, Lu SN, et al. Risk of hepatocellular carcinoma across a biological gradient of serum hepatitis $B$ virus DNA level. JAMA 2006;295:65-73.

17. Kumada T, Toyoda H, Tada T, Kiriyama S, Tanikawa M, Hisanaga $Y$, et al. Effect of nucleos(t)ide analogue therapy on hepatocarcinogenesis in chronic hepatitis B patients: a propensity score analysis. J Hepatol 2013;58:427-433.

18. Papatheodoridis GV, Manolakopoulos S, Touloumi G, Nikolopoulou G, Raptopoulou-Gigi M, Gogos C, et al. Hepatocellular carcinoma risk in $\mathrm{HBeAg}$-negative chronic hepatitis $\mathrm{B}$ patients with or without cirrhosis treated with entecavir: HepNet.Greece cohort. J Viral Hepat 2015;22:120-127.

19. Lim YS, Han S, Heo NY, Shim JH, Lee HC, Suh DJ. Mortality, liver transplantation, and hepatocellular carcinoma among patients with chronic hepatitis B treated with entecavir vs lamivudine. Gastroenterology 2014;147:152-161.

20. Chang TT, Liaw YF, Wu SS, Schiff E, Han KH, Lai CL, et al. Long-term entecavir therapy results in the reversal of fibrosis/cirrhosis and continued histological improvement in patients with chronic hepatitis $B$. 
Hepatology 2010;52:886-893.

21. Marcellin P, Gane E, Buti M, Afdhal N, Sievert W, Jacobson IM, et al. Regression of cirrhosis during treatment with tenofovir disoproxil fumarate for chronic hepatitis B: a 5-year open-label follow-up study. Lancet 2013;381:468-475.

22. Wong GL, Chan HL, Chan HY, Tse PC, Tse YK, Mak CW, et al. ACcuracy of risk scores for patients with chronic hepatitis $B$ receiving entecavir treatment. Gastroenterology 2013;144:933-944.

23. Jung KS, Kim SU, Song K, Park JY, Kim DY, Ahn SH, et al. Validation of hepatitis $B$ virus-related hepatocellular carcinoma prediction models in the era of antiviral therapy. Hepatology 2015;62:1757-
1766.

24. Cho JH, Yoon KH, Lee KE, Park DS, Lee YJ, Moon HB, et al. Distribution of hepatitis $B$ virus genotypes in Korea. Korean J Hepatol 2009;15:140-147.

25. Yang HI, Yeh SH, Chen PJ, Iloeje UH, Jen CL, Su J, et al. Associations between hepatitis $B$ virus genotype and mutants and the risk of hepatocellular carcinoma. J Natl Cancer Inst 2008;100:1134-1143.

26. European Association For The Study Of The Liver; European Organisation For Research And Treatment Of Cancer. EASL-EORTC clinical practice guidelines: management of hepatocellular carcinoma. J Hepatol 2012;56:908-943. 